\title{
SEMIGROUPS OF OPERATORS THAT DESCRIBE A FELLER PROCESS ON THE LINE, WHICH IS THE RESULT OF PASTING TOGETHER TWO DIFFUSION PROCESSES
}

UDC 519.21

\author{
P. P. KONONCHUK AND B. I. KOPYTKO
}

\begin{abstract}
We use the method of the classical potential theory to construct the semigroup of operators that describe a Feller process on the line by pasting together two diffusion processes that satisfy a nonlocal Feller-Wentzell type condition for the pasting.
\end{abstract}

\section{Main Notation And Setting of the PRoblem}

Let $D_{i}=\left\{x:(-1)^{i} x>0\right\}, i=1,2$, be two domains on the real line, let $\partial D=\{0\}$ be the joint boundary for these domains, and let $\overline{D_{i}}=D_{i} \cup \partial D$ be the closure of $D_{i}$. If $D$ is a domain in $\mathbb{R}$ (in particular, $D=\mathbb{R}$ ) and $\bar{D}$ is its closure, then $C_{b}(\bar{D})$ denotes the Banach space of bounded continuous functions $\varphi(x)$ on $\bar{D}$ equipped with the norm $\|\varphi\|=\sup _{x \in \bar{D}}|\varphi(x)|$ and $C_{0}(\bar{D})$ denotes the space of bounded uniformly continuous functions on $\bar{D}$.

In what follows, if $\varphi \in C_{b}(\mathbb{R})$, then the restriction of $\varphi$ to $\bar{D}_{i}$ is denoted by $\varphi_{i}$. Let $C_{2}\left(\bar{D}_{i}\right)$ be the subset of $C_{b}\left(\bar{D}_{i}\right)$ containing all the functions $\varphi$ such that $\varphi_{i}, \varphi_{i}^{\prime}, \varphi_{i}^{\prime \prime} \in$ $C_{0}\left(\bar{D}_{i}\right), i=1,2$.

Assume that a differential operator $L_{i}$ is defined in the domain $D_{i}, i=1,2$, and that it acts on functions $\varphi$ of $C_{2}\left(\bar{D}_{i}\right)$,

$$
L_{i} \varphi(x)=\frac{1}{2} b_{i}(x) \frac{d^{2} \varphi_{i}}{d x^{2}}(x)+a_{i}(x) \frac{d \varphi_{i}}{d x}(x), \quad i=1,2,
$$

where $b_{i}(x)$ and $a_{i}(x)$ are bounded continuous functions on $\bar{D}_{i}$ and, moreover, $b_{i}(x) \geq 0$. We further assume that each operator $L_{i}$ generates a homogeneous diffusion process on $D_{i}$. Denote by $C_{2}(\mathbb{R})$ the subset of $C_{b}(\mathbb{R})$ consisting of the functions $\varphi(x)$ such that $\varphi_{i} \in C_{2}\left(\bar{D}_{i}\right), i=1,2$, and $L_{1} \varphi_{1}(0)=L_{2} \varphi_{2}(0)$. Now we introduce the operator $L$ acting on functions $\varphi$ of $C_{2}(\mathbb{R})$ according to the rule

$$
L \varphi(x)= \begin{cases}L_{1} \varphi(x) & \text { if } x \in \bar{D}_{1} \\ L_{2} \varphi(x) & \text { if } x \in \bar{D}_{2}\end{cases}
$$

Then we introduce the additional pasting condition at the point $x=0$ that reduces the operator $L$ (its closure, more precisely) to the infinitesimal operator of the Feller semigroup on $C_{0}(\mathbb{R})$. This condition is written as

$$
L_{0} \varphi(0) \equiv q_{1} \varphi^{\prime}(0-)-q_{2} \varphi^{\prime}(0+)+\int_{D_{1} \cup D_{2}}(\varphi(0)-\varphi(y)) \mu(d y)=0 .
$$

2010 Mathematics Subject Classification. Primary 60J60.

Key words and phrases. Diffusion processes, discontinuities of trajectories, analytic methods, method of potential. 
Here $q_{i}, i=1,2$, are nonnegative numbers and $\mu(\cdot)$ is a nonnegative measure on $D_{1} \cup D_{2}$ such that

$$
\lambda(\delta)=\int_{\left(D_{1} \cup D_{2}\right) \backslash D_{\delta}}|y| \mu(d y)<\infty, \quad \mu\left(D_{\delta}\right)<\infty,
$$

where $D_{\delta}=\{x \in \mathbb{R}:|x|>\delta>0\}$. It is assumed that not all numbers among $q_{1}, q_{2}$, and $m=\mu\left(D_{1} \cup D_{2}\right)$ equal 0 .

Note that (see [1]) condition (3) is a particular case of a more general Feller-Wentzell type condition for pasting $([2,3])$. Conditions of this type are used to describe the behavior of a diffusion process after it reaches the joint boundary of the domains $D_{i}$, $i=1,2$. In the case under consideration, the left hand side of (3) contains the terms corresponding to the partial reflection of the process at the point $x=0$ and those corresponding to its jumps to one of the domains $D_{1}$ or $D_{2}$. Besides the two types of behavior mentioned above, the diffusion process may have a delay or may disappear at the boundary. Thus two extra terms appear in the general Feller-Wentzell pasting condition.

We study the question of the existence of a semigroup of operators $\left\{\mathcal{T}_{t}\right\}_{t \geq 0}$ describing a Feller process (not necessarily continuous) that is defined on $\mathbb{R}$ and coincides with a diffusion process generated by the operator $L_{i}$ in $D_{i}, i=1,2$. The behavior of the process at the point $x=0$ is determined by the pasting condition (3). We consider the case where the parameters $q_{1}$ and $q_{2}$ in (3) satisfy the condition

$$
q_{1}+q_{2}>0
$$

The problem stated above is often called the problem on pasting together two diffusion processes on the real line (see [1, 4, 5, 6]).

We use analytical methods to solve this problem. When applying this approach (see [4, 5, 6]), the problem reduces to the corresponding problem of conjugation for a linear parabolic second order equation with discontinuous coefficients. The latter problem is to find a function $u(t, x)$ defined for $t>0$ and $x \in \mathbb{R}$ such that

$$
\begin{gathered}
\frac{\partial u}{\partial t}(t, x)=L_{i} u(t, x), \quad t>0, x \in D_{i}, i=1,2, \\
u(0, x)=\varphi(x), \quad x \in \mathbb{R}, \\
u(t, 0-)=u(t, 0+), \quad t \geq 0, \\
L_{0} u(t, 0)=0, \quad t>0,
\end{gathered}
$$

where $\varphi \in C_{b}(\mathbb{R})$ is a given function.

Conjugation condition (8) in the problem (6) -(9) reflects the Feller property of the semigroup to be found, while equality (9) corresponds to the pasting condition (3).

We prove that problem (6) - (9) has a solution under some additional assumptions imposed on the coefficients of the operators $L_{i}, i=1,2$. The proof applies the method of limit integral equations and uses an ordinary fundamental solution of a parabolic equation and heat potentials generated by this equation. A similar problem is studied in [4] by using the potential method if $m=0$. The case of $q_{1}=q_{2}=0$ is considered in [6] for a finite measure $\mu(\cdot)$. In the paper [7], the Markov process constructed by pasting together two Brownian motions is obtained in a different way if condition (3) does not contain the integral term and if $q_{1}=q_{2}$ (this is the so-called classical case of pasting together two diffusion processes).

In what follows let $T$ be a fixed positive number, $\mathbb{R}_{T}^{2} \equiv(0, T] \times \mathbb{R}$,

$$
\mathbb{R}_{\infty}^{2} \equiv(0, \infty) \times \mathbb{R}
$$


let $D_{t}^{r}\left(D_{x}^{p}\right)$ be the symbol of the derivative (partial derivative) of order $r$ (of order $p$ ) with respect to the variable $t(x)$, where $r$ and $p$ are nonnegative integers; let $C^{m, l}(\Omega)$ $\left(C^{m, l}(\bar{\Omega})\right), m=0,1, l=0,1,2$, be the family of continuous functions in $\Omega$ (in $\bar{\Omega}$ ) whose derivatives $D_{t}^{r}, D_{x}^{p}, r \leq m, p \leq l$, are continuous in $\Omega(\bar{\Omega})$, where $\Omega$ is a subset of $\mathbb{R}_{\infty}^{2}$; let $H^{\alpha}(\mathbb{R}), \alpha \in(0,1)$, denote the Hölder space (see [8]). Here

$$
C^{0,0}(\Omega) \equiv C(\Omega) \quad \text { and } \quad C^{0,0}(\bar{\Omega}) \equiv C(\bar{\Omega}) .
$$

Throughout the paper $C$ and $c$ denote those constants whose precise values do not matter for our reasoning. These constants may be different in different places but do not depend on $(t, x)$. Other notation will be introduced in appropriate places.

\section{Fundamental solution of a SECONd order PARABolic EQUATion, AND RELATED POTENTIALS}

Consider parabolic operators $D_{t}-L_{i}, i=1,2$, in the domain $\mathbb{R}_{\infty}^{2}$. Without loss of generality, assume that the coefficients of these operators are defined in $\mathbb{R}$ and satisfy the following conditions:

a) $b_{i}, a_{i} \in H^{\alpha}(\mathbb{R}), i=1,2$;

b) there are positive constants $b_{i 0}, i=1,2$, such that $b_{i}(x) \geq b_{i 0}$ for all $x \in \mathbb{R}$.

Let $\left.g_{i}(t, x, y)\right), t>0, x, y \in \mathbb{R}$, be the fundamental solutions of the operators $D_{t}-L_{i}$, $i=1,2$ (see [5, Chapter II, $\S 2]$ or [8, Chapter IV, $\S 11])$ :

$$
g_{i}(t, x, y)=g_{i 0}(t, x, y)+g_{i 1}(t, x, y), \quad i=1,2,
$$

where

$$
g_{i 0}(t, x, y)=\left(2 \pi b_{i}(y) t\right)^{-1 / 2} \exp \left(-\frac{(y-x)^{2}}{2 b_{i}(y) t}\right),
$$

and the $g_{i 1}$ are integral terms that have a weaker singularity as $t \rightarrow 0$ than the $g_{0 i}$ do; moreover $g_{i}(t, x, y)=0$ for $t \leq 0$. Recall that the functions $g_{i}(t, x, y), i=1,2$, are continuous and nonnegative with respect to all arguments and satisfy equation (6) in the domain $(t, x) \in \mathbb{R}_{\infty}^{2}$ if $y$ is fixed. Further,

$$
\lim _{t \downarrow 0} \int_{\mathbb{R}} g_{i}(t, x, y) \varphi(y) d y=\varphi(x), \quad i=1,2,
$$

for $x \in \mathbb{R}$ and $\varphi \in C_{b}(\mathbb{R})$.

If $2 r+p \leq 2$, then

$$
\left|D_{t}^{r} D_{x}^{p} g_{i}(t, x, y)\right| \leq C t^{-(1+2 r+p) / 2} \exp \left(-c \frac{(y-x)^{2}}{t}\right), \quad t \in(0, T],
$$

and

$$
\left|D_{t}^{r} D_{x}^{p} g_{i 1}(t, x, y)\right| \leq C t^{-(1+2 r+p-\alpha) / 2} \exp \left(-c \frac{(y-x)^{2}}{t}\right), \quad t \in(0, T]
$$


for $i=1,2$ and $x, y \in \mathbb{R}$. We have

$$
\begin{gathered}
\int_{\mathbb{R}} g_{i}(t, x, y) d y=1 \\
\int_{\mathbb{R}} g_{i}(t, x, y)(y-x) d y=\int_{0}^{t} d \tau \int_{\mathbb{R}} g_{i}(\tau, x, y) a_{i}(y) d y \\
\int_{\mathbb{R}} g_{i}(t, x, y)(y-x)^{2} d y \\
=\int_{0}^{t} d \tau \int_{\mathbb{R}} g_{i}(\tau, x, y) b_{i}(y) d y+2 \int_{0}^{t} d \tau \int_{\mathbb{R}} g_{i}(\tau, x, y) a_{i}(y)(y-x) d y
\end{gathered}
$$

for $i=1,2, t>0$, and $x \in \mathbb{R}$.

Consider the following integrals:

$$
\begin{gathered}
u_{i 0}(t, x)=\int_{\mathbb{R}} g_{i}(t, x, y) \varphi(y) d y, \quad i=1,2, \\
u_{i 1}(t, x)=\int_{0}^{t} g_{i}(t-\tau, x, 0) V_{i}(\tau) d \tau, \quad i=1,2 .
\end{gathered}
$$

Here $\varphi(x)$ and $V_{i}(t), i=1,2$, are given functions. The functions $u_{i 0}$ and $u_{i 1}$ are called the Poisson potential and a simple layer potential, respectively, in the theory of parabolic equations. The definition and main properties of the fundamental solution $g_{i}, i=1,2$, imply that if $\varphi \in C_{b}(\mathbb{R})$, then the functions $u_{i 0}(t, x)$ are continuous in $\overline{\mathbb{R}_{\infty}^{2}}$, bounded with respect to $x$, and satisfy equation (6) in the domain $(t, x) \in \mathbb{R}_{\infty}^{2}$ and the initial condition (7). Moreover,

$$
\left|D_{t}^{r} D_{x}^{p} u_{i 0}(t, x)\right| \leq C\|\varphi\| t^{-(2 r+p) / 2}, \quad 2 r+p \leq 2, i=1,2,
$$

in $\mathbb{R}_{T}^{2}$.

If the functions $V_{i}(t), i=1,2$, in (15) are bounded and continuous in $[0, \infty)$, then the functions $u_{i 1}(t, x)$ are continuous in $\overline{\mathbb{R}_{\infty}^{2}}$, bounded with respect to the argument $x$, satisfy equation ([6) in the domains $(t, x) \in(0, \infty) \times D_{1}$ and $(t, x) \in(0, \infty) \times D_{2}$, and the initial condition $u_{i 1}(0, x)=0, x \in \mathbb{R}, i=1,2$, holds.

Note another important property of the simple layer potential $u_{i 1}(t, x)$ concerning the behavior of its derivatives with respect to the argument $x$ in a neighborhood of the point $x=0$. The so-called jump formula is known in this case (see [5, Chapter II, §5], [8, Chapter IV, §15]). For the case under consideration, this result is written as

$$
D_{x} u_{i 1}(t, 0 \pm)=\int_{0}^{t} D_{x} g_{i 1}(t-\tau, 0,0) V_{i}(\tau) d \tau \mp \frac{V_{i}(t)}{b_{i}(0)}, \quad t>0, \quad i=1,2 .
$$

The existence of the integral in (17) follows from inequality (12) with $r=0, p=1$, and $x=y=0$.

The properties of the simple layer potential mentioned above hold under even more general assumptions concerning the functions $V_{i}, i=1,2$, in the integrals on the right hand side of (15).

\section{Solution of the Parabolic Problem of CONJugation}

Consider the problem (6) - (9). First we prove that a solution $u(t, x)$ exists. We search for a solution represented as a sum of two potentials,

$$
u(t, x)=u_{i 0}(t, x)+u_{i 1}(t, x), \quad(t, x) \in[0, \infty) \times \overline{D_{i}}, \quad i=1,2,
$$

where the functions $u_{i 0}$ and $u_{i 1}$ are defined by (14) and (15), respectively, and where the densities $V_{i}, i=1,2$, are unknown functions. To find the densities, we use the 
conjugation conditions (8) and (9). Substituting the right hand sides of relations (18) into equalities (8) and (9) and taking (17) into account, we obtain the following system of integral equations with respect to $V_{i}, i=1,2$ :

$$
\begin{gathered}
\sum_{i=1}^{2} \int_{0}^{t}(-1)^{i-1} g_{i}(t-\tau, 0,0) V_{i}(\tau) d \tau=\Phi(t), \quad t>0, \\
\sum_{i=1}^{2}\left(\frac{q_{i}}{b_{i}(0)} V_{i}(t)-\int_{0}^{t} K_{i}(t-\tau) V_{i}(\tau) d \tau\right)=\Psi(t), \quad t>0,
\end{gathered}
$$

where

$$
\begin{gathered}
\Phi(t)=u_{20}(t, 0)-u_{10}(t, 0), \\
\Psi(t)=\sum_{i=1}^{2}\left((-1)^{i} q_{i} D_{x} u_{i 0}(t, 0)+\int_{D_{i}}\left(u_{i 0}(t, y)-u_{i 0}(t, 0)\right) \mu(d y)\right), \\
K_{i}(t-\tau)=(-1)^{i} q_{i} D_{x} g_{i 1}(t-\tau, 0,0)+\int_{D_{i}}\left(g_{i}(t-\tau, y, 0)-g_{i}(t-\tau, 0,0)\right) \mu(d y), \\
i=1,2 .
\end{gathered}
$$

The first equation in the system of integral equations (19) is the Volterra equation of the first kind, while the second is the Volterra equation of the second kind. Following Holmgren's idea [9], we reduce the Volterra equation of the first kind to the Volterra equation of the second kind. First we introduce the integro-differential operator $\mathcal{E}$ whose action is given by

$$
\mathcal{E}(t) \Phi=\sqrt{\frac{2}{\pi}} \frac{d}{d t} \int_{0}^{t}(t-s)^{-1 / 2} \Phi(s) d s, \quad t>0 .
$$

Denote the function on the right hand side of (20) by $\Phi_{0}(t)$. Since the function $\Phi(t)$ in (19) is continuously differentiable for $t>0$, we easily check that the right hand side of (20) can be written as

$$
\Phi_{0}(t) \equiv \mathcal{E}(t) \Phi=\frac{1}{\sqrt{2 \pi}} \int_{0}^{t}(t-s)^{-3 / 2}(\Phi(t)-\Phi(s)) d s+\sqrt{\frac{2}{\pi}} \Phi(t) t^{-1 / 2}, \quad t>0 .
$$

Moreover,

$$
\left|\Phi_{0}(t)\right| \leq C\|\varphi\| t^{-1 / 2}
$$

for $t \in(0, T]$, where $T$ is an arbitrary number.

Indeed, inequality (22) for the second term on the right hand isde of (21) follows from the bound (16) with $r=p=0$. To estimate the integral on the right hand side of (21), we split it into two parts, namely

$$
\int_{0}^{t}=\int_{0}^{t / 2}+\int_{t / 2}^{t}
$$

Applying estimate (16) for integrals involving the function $\Phi$ and the Lagrange mean value theorem for the difference $\Phi(t)-\Phi(s)$ in the second integral, we prove inequality (22) for the integral term in (21), whence this bound follows for $\Phi_{0}(t)$. Note that the function $\Psi(t)$ in (19) satisfies inequality (22), too. This follows from the mean value theorem for $u_{i 0}(t, y)-u_{i 0}(t, 0), i=1,2$, bounds (16), and condition (4). However, the constant $C$ in (22) depends on $T$ and on $\delta$ in this case.

A simple algebra checks that the operator $\mathcal{E}$ applied to both sides of the first equation in (19) transforms it to the Volterra integral equation of the second kind. This changes 
the system of equations (19) to the equivalent system of two integral equations with respect to $V_{i}, i=1,2$, namely

$$
V_{i}(t)=\sum_{j=1}^{2} \int_{0}^{t} K_{i j}(t-\tau) V_{j}(\tau) d \tau+\Psi_{i}(t), \quad t>0, \quad i=1,2,
$$

where

$$
\begin{gathered}
\Psi_{i}(t)=d_{i}\left(\Psi(t)+\frac{(-1)^{i+1} q_{3-i}}{\left.\sqrt{b_{3-i}(0)} \Phi_{0}(t)\right), \quad i=1,2,}\right. \\
K_{i j}(t-\tau)=d_{i}\left(K_{j}(t-\tau)+\frac{(-1)^{i-1} q_{3-i}}{\sqrt{b_{3-i}(0)}} R_{j}(t-\tau)\right), \quad i, j=1,2, \\
d_{i}=\frac{\sqrt{b_{i}(0)}}{\frac{q_{1}}{\sqrt{b_{1}(0)}}+\frac{q_{2}}{\sqrt{b_{2}(0)}}, \quad i=1,2,} \\
R_{j}(t-\tau)=\frac{(-1)^{j}}{\sqrt{2 \pi}}\left(\int_{\tau}^{t}(t-s)^{-3 / 2}\left(g_{j 1}(t-\tau, 0,0)-g_{j 1}(s-\tau, 0,0)\right) d s\right. \\
\left.+2(t-\tau)^{-1 / 2} g_{j 1}(t-\tau, 0,0)\right), \quad j=1,2 .
\end{gathered}
$$

The properties of $\Psi(t)$ and $\Phi_{0}(t)$ imply that $\Psi_{i}(t), i=1,2$, are continuous functions for $t>0$ and satisfy the following bound:

$$
\left|\Psi_{i}(t)\right| \leq M(\delta)\|\varphi\| t^{-1 / 2}, \quad i=1,2,
$$

where $t \in(0, T]$ and $M(\delta)$ is a constant.

Now we study the kernels in the integral equations (23). Let $D_{j, \delta}=\left\{y \in D_{j}:|y|>\delta\right\}$, $j=1,2$. Using (10) we represent $K_{i j}$ as follows:

$$
\begin{aligned}
K_{i j}(t-\tau) & =d_{i}\left(K_{j}^{(1)}(t-\tau)+\frac{(-1)^{i+1} q_{3-i}}{\sqrt{b_{3-i}(0)}} R_{j}(t-\tau)\right)+d_{i} K_{j}^{(2)}(t-\tau) \\
& =K_{i j}^{(1)}(t-\tau)+K_{i j}^{(2)}(t-\tau), \quad i, j=1,2
\end{aligned}
$$

where

$$
\begin{aligned}
& K_{j}^{(1)}(t-\tau)=(-1)^{j} q_{j} D_{x} g_{j 1}(t-\tau, 0,0)+\int_{D_{j, \delta}}\left(g_{j}(t-\tau, y, 0)-g_{j}(t-\tau, 0,0)\right) \mu(d y) \\
&+\int_{D_{j} \backslash D_{j, \delta}}\left(g_{j 1}(t-\tau, y, 0)-g_{j 1}(t-\tau, 0,0)\right) \mu(d y), \\
& K_{j}^{(2)}(t-\tau)=\int_{D_{j} \backslash D_{j, \delta}}\left(g_{j 0}(t-\tau, y, 0)-g_{j 0}(t-\tau, 0,0)\right) \mu(d y) .
\end{aligned}
$$

Using the mean value theorem for $g_{j 1}(t-\tau, y, 0)-g_{j 1}(t-\tau, 0,0)$, the bounds (11) and (12), and condition (44), we obtain the inequality

$$
\left|K_{i j}^{(1)}(t-\tau)\right| \leq N(\delta)(t-\tau)^{-1+\alpha / 2}, \quad i, j=1,2,
$$

for $0 \leq \tau<t \leq T$ and some constant $N(\delta)$.

Following the same method for the integral $K_{j}^{(2)}(t-\tau)$, we get

$$
\left|K_{i j}^{(2)}(t-\tau)\right| \leq C(t-\tau)^{-1}
$$


This inequality means that the corresponding part of every kernel $K_{i j}$ has a nonintegrable singularity. Nevertheless, we prove that the method of sequential approximations applies to the system (23).

We find a solution of the system (23) such that

$$
V_{i}(t)=\sum_{k=0}^{\infty} V_{i}^{(k)}(t), \quad i=1,2,
$$

where

$$
\begin{gathered}
V_{i}^{(0)}(t)=\Psi_{i}(t), \quad i=1,2, \\
V_{i}^{(k)}(t)=\sum_{j=1}^{2} \int_{0}^{t} K_{i j}(t-\tau) V_{j}^{(k-1)}(\tau) d \tau, \quad i=1,2, \quad k \in \mathbb{N} .
\end{gathered}
$$

First we estimate $V_{i}^{(1)}(t)$. Applying (25), we obtain

$V_{i}^{(1)}=\sum_{j=1}^{2} \int_{0}^{t} K_{i j}^{(1)}(t-\tau) \Psi_{j}(\tau) d \tau+\sum_{j=1}^{2} \int_{0}^{t} K_{i j}^{(2)}(t-\tau) \Psi_{j}(\tau) d \tau=I_{i 1}+I_{i 2}, \quad i=1,2$.

The first integral on the right hand side is estimated with the help of inequalities (26) and (27). Indeed,

$$
\begin{aligned}
\left|I_{i 1}\right| & \leq M(\delta)\|\varphi\| 2 N(\delta) \int_{0}^{t}(t-\tau)^{-1+\alpha / 2} \tau^{-1 / 2} d \tau \\
& =M(\delta)\|\varphi\| \frac{2 N(\delta) \Gamma\left(\frac{\alpha}{2}\right) \Gamma\left(\frac{1}{2}\right)}{\Gamma\left(\frac{1}{2}+\frac{\alpha}{2}\right)} t^{-1 / 2+\alpha / 2}, \quad t \in(0, T], \quad i=1,2 .
\end{aligned}
$$

To estimate the second term $I_{i 2}$, we use the equalities

$$
\begin{gathered}
g_{j 0}(t-\tau, y, 0)-g_{j 0}(t-\tau, 0,0)=\frac{1}{\sqrt{2 \pi b_{j}(0)(t-\tau)}} \int_{0}^{1} \frac{d}{d \Theta} \exp \left(-\frac{y^{2} \Theta}{2 b_{j}(0)(t-\tau)}\right) d \theta \\
\int_{0}^{t}(t-\tau)^{-3 / 2} \tau^{-1 / 2} \exp \left(-\frac{y^{2} \Theta}{2 b_{j}(0)(t-\tau)}\right) d \Theta=\frac{\sqrt{2 \pi b_{j}(0)}}{|y| \sqrt{\theta t}} \exp \left(-\frac{y^{2} \Theta}{2 b_{j}(0) t}\right)
\end{gathered}
$$

for $j=1,2$. Then we use estimate (24) and the inequality

$$
\frac{d_{i}}{b_{j}(0)} \leq d=\frac{b^{+}(0)}{b^{-}(0)\left(q_{1}+q_{2}\right)}, \quad i, j=1,2,
$$

where $b^{+}(0)=\max \left(b_{1}(0), b_{2}(0)\right)$ and $b^{-}(0)=\min \left(b_{1}(0), b_{2}(0)\right)$. As a result, we get

$$
\begin{aligned}
\left|I_{i 2}\right| \leq & M(\delta)\|\varphi\| d_{i} \sum_{j=1}^{2} \frac{1}{2 \sqrt{2 \pi}\left(b_{j}(0)\right)^{3 / 2}} \int_{D_{j} \backslash D_{j, \delta}} y^{2} \mu(d y) \\
& \times \int_{0}^{1} d \Theta \int_{0}^{t}(t-\tau)^{-3 / 2} \tau^{-1 / 2} \exp \left(-\frac{y^{2} \Theta}{2 b_{j}(0)(t-\tau)}\right) d \tau \\
\leq & M(\delta)\|\varphi\| t^{-1 / 2} d \sum_{j=1}^{2} \int_{D_{j} \backslash D_{j, \delta}}|y| \mu(d y) \\
= & M(\delta)\|\varphi\| t^{-1 / 2} d \lambda(\delta), \quad t \in(0, T], \quad i=1,2 .
\end{aligned}
$$

Now consider $d \lambda(\delta)$. Condition (4) implies that $\lambda(\delta) \rightarrow 0$ as $\delta \rightarrow 0$. This allows one to choose a number $\delta=\delta_{0}$ such that $d_{0}=d \lambda\left(\delta_{0}\right)<1$. In what follows, all the constants 
depending on $\delta$ are considered for $\delta=\delta_{0}$. Combining the estimates (28) and (29), we get

$$
\left|V_{i}^{(1)}(t)\right| \leq M\left(\delta_{0}\right)\|\varphi\| t^{-1 / 2}\left(\frac{2 N\left(\delta_{0}\right) \Gamma\left(\frac{\alpha}{2}\right) \Gamma\left(\frac{1}{2}\right)}{\Gamma\left(\frac{1+\alpha}{2}\right)} t^{\alpha / 2}+d_{0}\right), \quad i=1,2,
$$

for $t \in(0, T]$. Let $M=M\left(\delta_{0}\right), N=N\left(\delta_{0}\right)$, and

$$
h_{t}=\frac{2 N \Gamma\left(\frac{\alpha}{2}\right) \Gamma\left(\frac{1}{2}\right)}{\Gamma\left(\frac{1+\alpha}{2}\right)} t^{\alpha / 2}
$$

in the right hand side of (30). Using the induction in $k$ for $V_{i}^{(k)}(t), t \in(0, T], i=1,2$, we prove the estimate

$$
\left|V_{i}^{(k)}(t)\right| \leq M\|\varphi\| t^{-1 / 2} \sum_{m=0}^{k}\left(\begin{array}{l}
n \\
k
\end{array}\right) h_{t}^{(k-n)} d_{0}^{n}, \quad k=0,1, \ldots,
$$

where

This implies that

$$
h_{t}^{(k)}=\frac{\left(2 N \Gamma\left(\frac{\alpha}{2}\right)\right)^{k} \Gamma\left(\frac{1}{2}\right)}{\Gamma\left(\frac{1+k \alpha}{2}\right)} t^{k \alpha / 2}
$$

$$
\begin{aligned}
\sum_{k=0}^{\infty}\left|V_{i}^{(k)}(t)\right| & \leq M\|\varphi\| t^{-1 / 2} \sum_{k=0}^{\infty} \sum_{n=0}^{k}\left(\begin{array}{c}
n \\
k
\end{array}\right) h_{t}^{(k-n)} d_{0}^{n} \\
& =M\|\varphi\| t^{-1 / 2} \sum_{k=0}^{\infty} h_{t}^{(k)} \sum_{n=0}^{\infty}\left(\begin{array}{c}
k+n \\
n
\end{array}\right) d_{0}^{n} \\
& =M\|\varphi\| t^{-1 / 2} \sum_{k=0}^{\infty} \frac{h_{t}^{(k)}}{\left(1-d_{0}\right)^{k+1}} \\
& =M\|\varphi\| t^{-1 / 2} \sum_{k=0}^{\infty} \frac{\left(2 N \Gamma\left(\frac{\alpha}{2}\right)\right)^{k} \Gamma\left(\frac{1}{2}\right)}{\Gamma\left(\frac{1+k \alpha}{2}\right)\left(1-d_{0}\right)^{k+1}} t^{k \alpha / 2}
\end{aligned}
$$

for $t \in(0, T]$ and $i=1,2$. Inequality (31) implies the convergence of series (27) for $t>0$ and the bound

$$
\left|V_{i}(t)\right| \leq c\|\varphi\| t^{-1 / 2}, \quad i=1,2
$$

where $t \in(0, T]$ and $C$ is a constant.

Estimates (11) for $r=p=0$ and (32) prove that the integrals on the right hand side of (15) exist and that

$$
\left|u_{i 1}(t, x)\right| \leq C\|\varphi\|, \quad i=1,2
$$

in the domain $\mathbb{R}_{T}^{2}$.

A further analysis of series (27) and integrals in (15) shows that the functions $u_{i 1}(t, x)$ are continuous for $t=0$ and $x \in \mathbb{R}$ as well, and that $u_{i 1}(0, x)=0, i=1,2$. Thus we conclude that the function $u(t, x)$ defined by relations (18) and (27) is a solution of the problem (6) - (9).

The proof of the uniqueness of a solution of the problem (6) - (9) follows the lines of the proof of an analogous result in [6].

The results proved above are stated below.

Theorem 3.1. Let the coefficients of the operators $L_{i}, i=1,2$, in (1) satisfy assumptions a) and b) (in $\S 2$ ), while the parameters $q_{1}, q_{2}$, and the measure $\mu(\cdot)$ in (3) satisfy conditions (5) and (4), respectively. Then the problem (6) -(9) has a unique solution

$$
u \in C\left(\overline{\mathbb{R}_{\infty}^{2}}\right) \cap C^{1,2}\left((0, \infty) \times D_{i}\right), \quad i=1,2
$$


for all functions $\varphi \in C_{b}(\mathbb{R})$. Moreover

$$
|u(t, x)| \leq C\|\varphi\|, \quad(t, x) \in \overline{\mathbb{R}_{T}^{2}} .
$$

Theorem 3.2. A solution of the problem (6) -(9) that belongs to the class (34) is the sum of potentials (14) and (15), where $V_{i}, i=1,2$, is a solution of the system of integral equations (23).

\section{Construction of the Process}

Theorems 3.1 and 3.2 imply that a family of operators $\left(\mathcal{T}_{t}\right)_{t \geq 0}$ can be defined with the help of a solution of the problem (6) - (9) (the operators act in the space $C_{b}(\mathbb{R})$ ). For $t>0, x \in \mathbb{R}$, and $\varphi \in C_{b}(\mathbb{R})$ put

$$
\mathcal{T}_{t} \varphi(x)=\int_{\mathbb{R}} g_{i}(t, x, x y) \varphi(y) d y+\int_{0}^{t} g_{i}(t-\tau, x, 0) V_{i}(\tau, \varphi) d \tau
$$

for $t>0, x \in \overline{D_{i}}$, and $i=1,2$, where $V_{i}(t, \varphi) \equiv V_{i}(t), i=1,2$, is a solution of the system of integral equations (23) defined by relation (27). Note that $\mathcal{T}_{0}=I$, where $I$ is the unit operator, and that $\mathcal{T}_{t} \varphi(x)$ admits bound (35) in the domain $(t, x) \in \overline{\mathbb{R}_{T}^{2}}$.

The above integral representation for the family of operators $\left(\mathcal{T}_{t}\right)_{t \geq 0}$ implies the following properties:

1) if $\varphi_{n} \in C_{b}(\mathbb{R}), n \in \mathbb{N}, \sup _{n}\left\|\varphi_{n}\right\|<\infty$, and if $\lim _{n \rightarrow \infty} \varphi_{n}(x)=\varphi(x)$ for all $x \in \mathbb{R}$, where $\varphi \in C_{b}(\mathbb{R})$, then for all $t \geq 0$ and $x \in \mathbb{R}$,

$$
\lim _{n \rightarrow \infty} \mathcal{T}_{t} \varphi_{n}(x)=\mathcal{T}_{t} \varphi(x) ;
$$

2) for all $t_{1} \geq 0$ and $t_{2} \geq 0$,

$$
\mathcal{T}_{t_{1}+t_{2}}=\mathcal{T}_{t_{1}} \cdot \mathcal{T}_{t_{2}}
$$

3) $\mathcal{T}_{t} \varphi(x) \geq 0$ for all $t \geq 0$ and $x \in \mathbb{R}$ if $\varphi \in C_{b}(\mathbb{R})$ and $\varphi(x) \geq 0$;

4) $\left\|\mathcal{T}_{t}\right\| \leq 1$ for all $t \geq 0$.

We outline the proof of these properties. Property 1) follows from the equality

$$
\lim _{n \rightarrow \infty} V_{i}\left(t, \varphi_{n}\right)=V_{i}(t, \varphi), \quad t>0, i=1,2,
$$

and from the Lebesgue dominated convergence theorem. Property 2), called the semigroup property of operators $\mathcal{T}_{t}$, follows from the uniqueness of a solution of the problem (6) - (9) proved in Theorem 3.1. Property 3) means that the cone of nonnegative functions is invariant under the semigroup $\mathcal{T}_{t}$. The proof of this property is the same as that of a lemma in [4 (see also [5, p. 82]) and uses the maximum principle for parabolic equations. Finally, property 4 ) means that $\mathcal{T}_{t}$ is a contraction operator for all $t \geq 0$. To prove this property, we note that $\mathcal{T}_{t} \varphi_{0}(x) \equiv 1$ for all $t \geq 0$ and $x \in \mathbb{R}$ if $\varphi_{0}(x) \equiv 1$; then we use property 3 ).

Properties 1)-4) imply that the semigroup of operators $T_{t}, t \geq 0$, constructed by using equalities (36) and (27), defines a homogeneous Feller process. The transition probabilities of this process are denoted by $P(t, x, d y)$. Thus

$$
\mathcal{T}_{t} \varphi(x)=\int_{\mathbb{R}} P(t, x, d y) \varphi(y) .
$$

Therefore we proved the following result.

Theorem 4.1. The semigroup of operators defined by (36) and (27) generates a homogeneous Feller process in $\mathbb{R}$ that coincides at inner points of the domains $D_{1}$ and 
$D_{2}$ with the two given diffusion processes generated by the operators $L_{1}$ and $L_{2}$, respectively. The behavior of this Feller process at the point $x=0$ is determined by the pasting condition (3).

Remark 4.1. Conditions (44) and (5) imply that the construction of the Feller process presented in Theorem 4.1 also works for the case where $m=\mu\left(D_{1} \cup D_{2}\right)=0$. As noted above, this case is studied in the paper [4. In particular, it is shown in [4 that the trajectories of the constructed process are continuous and that it can be treated as a generalized diffusion process in the sense of Portenko [5]. This means that the diffusion coefficients for such a process are generalized functions of the Dirack $\delta$-function type concentrated at the point $x=0$ (more precisely, only the drift coefficient is a generalized function in this case).

We now turn back to the process constructed above. The presence of the integral term in the pasting condition (3) means that the trajectories of the process are, in general, discontinuous. On the other hand, condition (5) guarantees the positive probability that the original diffusion processes, after they pass through the point $x=0$, may continue without breaks in their trajectories, that is, continuously. A natural question arises about the influence of the jump measure of the process $\mu(\cdot)$ on the local behavior at the point $x=0$ of a diffusion particle moving along continuous trajectories. A partial answer to this question can be obtained by defining the generalized diffusion coefficients of the process constructed above. The existence of these characteristics can be proved under the condition that the measure $\mu(\cdot)$ in (3) satisfies the following additional conditions:

$$
\int_{D_{1} \cup D_{2}}|y| \mu(d y)<\infty, \quad \int_{D_{1} \cup D_{2}} y^{2} \mu(d y)<\infty .
$$

If conditions (38) hold, then equality (13) implies that

$$
\begin{aligned}
& \lim _{t \downarrow 0} \int_{\mathbb{R}} \varphi(x)\left(\frac{1}{t} \int_{\mathbb{R}}(y-x) P(t, x, d y)\right) d x=\int_{\mathbb{R}} a(x) \varphi(x) d x+a_{0} \varphi(0), \\
& \lim _{t \downarrow 0} \int_{\mathbb{R}} \varphi(x)\left(\frac{1}{t} \int_{\mathbb{R}}(y-x)^{2} P(t, x, d y)\right) d x=\int_{\mathbb{R}} b(x) \varphi(x) d x+b_{0} \varphi(0)
\end{aligned}
$$

for all continuous real-valued compactly supported functions $\varphi(x), x \in \mathbb{R}$, where

$$
\begin{aligned}
& a(x)= \begin{cases}a_{i}(x), & x \in D_{i}, i=1,2, \\
\sum_{i=1}^{2} l_{i} a_{i}(0), & x=0,\end{cases} \\
& b(x)= \begin{cases}b_{i}(x), & x \in D_{i}, i=1,2, \\
\sum_{i=1}^{2} l_{i} b_{i}(0), & x=0,\end{cases} \\
& l_{i}=\frac{q_{i} \sqrt{b_{3-i}(0)}}{q_{1} \sqrt{b_{2}(0)}+q_{2} \sqrt{b_{1}(0)}}, \quad i=1,2, \quad l_{1}+l_{2}=1, \\
& a_{0}=\frac{1}{2}\left(d_{1}+d_{2}\right)\left(q_{2}-q_{1}+m_{1}\right), \quad b_{0}=\frac{1}{2}\left(d_{1}+d_{2}\right) m_{2}, \\
& m_{1}=\int_{D_{1} \cup D_{2}} y \mu(d y), \quad m_{2}=\int_{D_{1} \cup D_{2}} y^{2} \mu(d y) .
\end{aligned}
$$

Equalities (39) mean that the diffusion coefficients exist in a generalized sense for the process constructed above; namely, there exists a diffusion coefficient $b(x)+b_{0} \delta(x)$ and drift coefficient $a(x)+a_{0} \delta(x)$, where $\delta(x)$ is the Dirack $\delta$-function.

The following result complements Theorem 4.1 
Theorem 4.2. Suppose the coefficients of the differential operators $L_{i}, i=1,2$, in (1) satisfy conditions a) and $b$ ). Assume further that the parameters $q_{i}, i=1,2$, and measure $\mu(\cdot)$ in (3) satisfy conditions (5) and (38), respectively. Then the semigroup of operators $\mathcal{T}_{t}, t \geq 0$, constructed by using equalities (36) and (27), generates a generalized diffusion process in $\mathbb{R}$ whose transition probability satisfies relation (39).

\section{BIBLIOGRAPHY}

1. H. Langer and W. Schenk, Knotting of one-dimensional Feller processes, Math. Nachr. 118 (1983), 151-161. MR725484 (85d:60139)

2. W. Feller, The parabolic differential equations and the associated semigroups of transformations, Ann. Math. 55 (1952), 468-519. MR0047886 (13:948a)

3. A. D. Ventcel' [Wentzell], Semigroups of operators corresponding to the generalized differential second order operator, Dokl. Akad. Nauk SSSR 111 (1956), no. 2, 269-272 (Russian) MR0092085 (19:1060c)

4. B. I. Kopytko, Pasting two diffusion processes on a line, Probabilistic methods of the infinitedimensional analysis, Institute of Mathematics, Academy of Sciences of Ukrain. SSR, Kiev, 1980, pp. 84-101. (Russian) MR623093 (82i:60126)

5. M. I. Portenko, Diffusion Processes in Media with Membranes, Institute of Mathematics, National Academy of Sciences of Ukraine, Kyiv, 1995. (Ukrainian) MR1356720 (96k:60200)

6. P. Kononchuk, Pasting of two diffusion processes on a line with nonlocal boundary conditions, Theory Stoch. Process. 14(30) (2008), no. 2, 52-59. MR2479733 (2010c:60227)

7. G. L. Kulinich, On the limit behavior of the distribution of the solution of a stochastic diffusion equation, Teor. Veroyatnost. Primenen. 12 (1967), no. 3, 548-551; English transl. in Theory Probab. Appl. 12 (1967), no. 3, 497-499. MR0215365 (35:6206)

8. O. A. Ladyzhenskaya, V. A. Solonnikov, and N. N. Ural'tseva, Linear and Quasilinear Equations of Parabolic Type, Nauka, Moscow, 1967; English transl., American Mathematical Society, Providence, Rhode Island, 1968. MR0241822 (39:3159b)

9. L. I. Kamynin, The existence of a solution of boundary value problems for a parabolic equation with discontinuous coefficients, Izvestiya Akad. Nauk SSSR, ser. matem. 28 (1964), no. 4, 721-744. (Russian) MR.0165245 (29:2534)

Department of Higher Mathematics, Faculty for Mechanics and Mathematics, L'viv National Ivan Franko University, Universytets'ka Street 1, L'viv 79000, Ukraine

E-mail address: p.kononchuk@gmail.com

Department of Higher Mathematics, Faculty for Mechanics and Mathematics, L'viv National Ivan Franko University, Universytets'Ka Street 1, L'viv 79000, Ukraine

E-mail address: bohdan.kopytko@gmail.com

Received 27/SEP/2010

Translated by N. SEMENOV 